

\title{
POTENCIAL SOLAR ACTIVO EN TECHUMBRES DE VIVIENDAS INMOBILIARIAS.
}

\section{THE POTENTIAL OF ACTIVE SOLAR SYSTEMS ON DWELLING ROOFS}

\author{
ESTEBAN FELIPE ZALAMEA LEÓN \\ Universidad de Cuenca \\ Cuenca, Ecuador \\ esteban.zalamea@ucuenca.edu.ec
}

\section{RESUMEN}

El presente artículo analiza las características geométricas de las techumbres de viviendas unifamiliares pertenecientes a conjuntos inmobiliarios recientes en Concepción (Chile) y su potencial de captación solar. Para ello, se ejecuta un registro de 2.139 casas construidas posteriores al año 2006, correspondientes a $68,3 \%$ del total. Al describir las cubiertas existentes, se detecta una superficie media de techumbre inclinada de $84,7 \mathrm{~m}^{2}$ y fragmentada, entre 3 y 12 alas o faldones, con inclinación media de $37,48^{\circ}$, que generan al menos un ala mayor o secundaria con superficie promedio es $33,9 \mathrm{~m} 2$ orientada al norte, este $\mathrm{u}$ oeste. Esta superficie recibe una radiación anual total de entre $954 \mathrm{kWha} / \mathrm{m}^{2}$ a $1.732,8 \mathrm{kWha} / \mathrm{m}^{2}$, según azimut e inclinación, lo que con paneles fotovoltaicos integrados produce potencialmente una media de 6.317,4 kWha. Además, se estudian casos extremos acorde a máxima y mínima superficie captadora por superficie construida, demostrando que la producción energética alcanzaría a abastecer eléctricamente con la tecnología fotovoltaica (PV) integrada. Finalmente, se evalúa el potencial de la tecnología híbrida térmica-fotovoltaica con fluido calotransportador de aire (PVTa).

\section{Palabras clave}

vivienda unifamiliar, potencial solar, techumbre, integración arquitectónica térmica fotovoltaica.

\section{ABSTRACT}

This paper presents an analysis of the geometric characteristics of roofs in recently-built, single family dwellings in housing developments in Concepcion, Chile, and their solar energy collection potential.

Data was recorded for 2,139 houses built after 2006, which corresponds to $68.28 \%$ of the total number of possible dwellings. It was found that the average surface area of sloped roofs is $84.7 \mathrm{~m}^{2}$ and is divided between three and twelve wings or gables, with an average incline of $37.48^{\circ}$. These result in at least one primary or secondary wing with an average surface of $33.9 \mathrm{~m} 2$ facing north, east or west. This surface receives a total annual solar radiation of from $954 \mathrm{kWha} / \mathrm{m}^{2}$ to $1,732.8 \mathrm{kWha/}$ $\mathrm{m}^{2}$ depending on the azimuth and the roof's slope. With the integration of photovoltaic panels, an average of 6,317.2 kWha per year, per house can potentially be produced. Additionally, when extreme cases were studied, including those with the largest and smallest roof surface areas, it was demonstrated that the energy produced would supply sufficient electricity with the integration of photovoltaic technology. Lastly, the potential of hybrid thermalphotovoltaic (PV/T) air technology is evaluated. 


\section{INTRODUCCIÓN.}

Lund (2012) y la International Energy Agency (2009) han expuesto cómo, en el futuro, ciudades enteras deberán incorporar masivamente energías renovables para reducir el alto impacto ambiental que supone su funcionamiento. Entre ellas, la captación solar activa juega un papel primordial, especialmente en zonas de latitudes medias. Para ello, es necesario caracterizar entornos urbanos, estableciendo potencialidades diversas frente a la demanda energética, con la finalidad de diseñar redes, almacenamiento y distribución adecuados. Un esquema urbanístico usual de ciudades en Chile, similar a muchos otros en el mundo, lo constituyen los centros urbanos densos, consolidados, con alta tasa de habitabilidad y usos variados: servicios administrativos, de comercio y habitacionales. Mientras, en los suburbios existe una menor densidad, con tendencia al uso habitacional y una tipología de casas unifamiliares. Dicha configuración está, en efecto, presente en Concepción.

Concepción es una ciudad costera localizada en la zona centro-sur de Chile y posee una población estimada de 230.730 habitantes (Observatorio Habitacional, 2014). Se encuentra en latitud media a $36,48^{\circ}$ Sur, presenta clima estacional, oscilaciones diarias e influencia marítima. La oferta inmobiliaria nos sugiere que la masa edificada nueva tipo casa predomina en el cinturón urbano externo, lo cual es fácilmente comprobable al comparar fotos satelitales secuenciales de distintos años recientes. Se levantan casas masivamente en forma repetitiva y seriada; y se ven modelos alternados iguales o similares, con techumbres siempre inclinadas. La relevancia de esta tipología está dada por su alta oferta y demanda, así como por las ventajas constructivas que representa la repetitividad.

La IEA (International Energy Agency, 2009) ha descrito condiciones de áreas urbanas que han de adoptar recolección solar activa de forma masiva. Wall et al. (2012), Munari (2009) y la IEA (IEA SHC Task 41 2012) en tanto, han analizado la situación actual y limitaciones para su implementación desde el ámbito arquitectónico. Hachem (2012), por su parte, ha propuesto una metodología para estimar el potencial solar de techumbres y barrios durante el proceso de diseño, no así para dimensionar emprendimientos ya existentes. Se han publicado, asimismo, alternativas para dimensionar el potencial solar a través de mapeo GIR (Izquierdo, Rodrigues y Fueyo, 2008) o por tomas aéreas con sobrevuelos LIDAR (Lukač y Žalik 2013), los cuales no consideran toda la techumbre, como tampoco el faldón o ala de techumbre con mayor aptitud por dimensión, orientación e inclinación, característica de las cubiertas inclinadas. La novedad del presente planteamiento consiste en dimensionar estadísticamente el ala de techumbre con mayor capacidad individual y, a la postre, dimensionar también su producción energética teórica en relación a la superficie construida y las demandas características promedio. Como se considera únicamente un faldón por caso y además las inclinaciones y la orientación (aunque de manera estadística), y como se adopta, asimismo, solamente una tipologia de casas en emprendimientos inmobiliarios, los resultados no son comparables con ningún otro caso detectado en la literatura especializada; no obstante, la menor variabilidad estacional y de irradiación que está presente en el contexto de estudio comparativamente con, el existente, por ejemplo, en el centro-norte de Europa, implica naturalmente una superior aptitud natural de Concepción, frente a aquellos sitios donde se ha desarrollado la recolección solar activa.

\section{METODOLOGÍA.}

Se delimitan zonas de la ciudad desarrolladas en años recientes $y$, seguidamente, se analiza el crecimiento desde finales de 2006 porque a partir de entonces están disponibles, a través de la herramienta temporal de GoogleEarth ${ }^{\circledR}$, fotos aéreas en alta resolución de la zona de estudio, lo que hace factible establecer comparaciones anuales de crecimiento, identificando los nuevos desarrollos y permitiendo a posteriori establecer la relación con los datos municipales de aprobaciones hasta 2013 (Observatorio Habitacional, 2015). Luego, se caracterizan los emprendimientos que ocupan más de $4000 \mathrm{~m}^{2}$, fácilmente identificables.

Se modela y dimensiona cada techumbre, se obtienen relaciones de ocupación, superficie de techumbre, superficie construida (en horizontal e inclinada), orientación, número de aguas, alas de techo potencialmente útiles, etc. Se precisa el Ala de cubierta de Mayor Dimensión (AMD), por modelo, aquella que posee mayor superficie con mayor potencial de captación; también la Segunda Ala de Mayor Dimensión (SAMD), como alternativa cuando la primera no esté bien orientada (Figura 1). Las pendientes se miden por caso; aspecto fundamental teniendo en cuenta, en primer lugar, que para la captación fotovoltaica la inclinación para maximizar la producción anual corresponde a aquella que coincide con la latitud o con pocos grados menos que ésta (hasta $10^{\circ}$ ), y, en segundo, que para captación térmica se supone una inclinación superior a la latitud (CDT, 2007; Hachem, 2012; Kalogirou, 2004).

Para obtener los indicadores principales se considera las características y parámetros dimensionales y la repetitividad de las viviendas. Después, se determina la irradiación factible mensual acorde a la inclinación y la orientación, y con desvíos previsibles, de acuerdo a datos recogidos de loteos a partir de modelo matemático. Tomando la irradiación horizontal global publicada en CDT (2007) y aplicando la fórmula [1] adoptada desde Ley Chilena 20365 (MINENERGIA, 2009), se obtiene el nivel de irradiación bajo distintas condiciones por $\mathrm{m}^{2}$, mensualmente: 

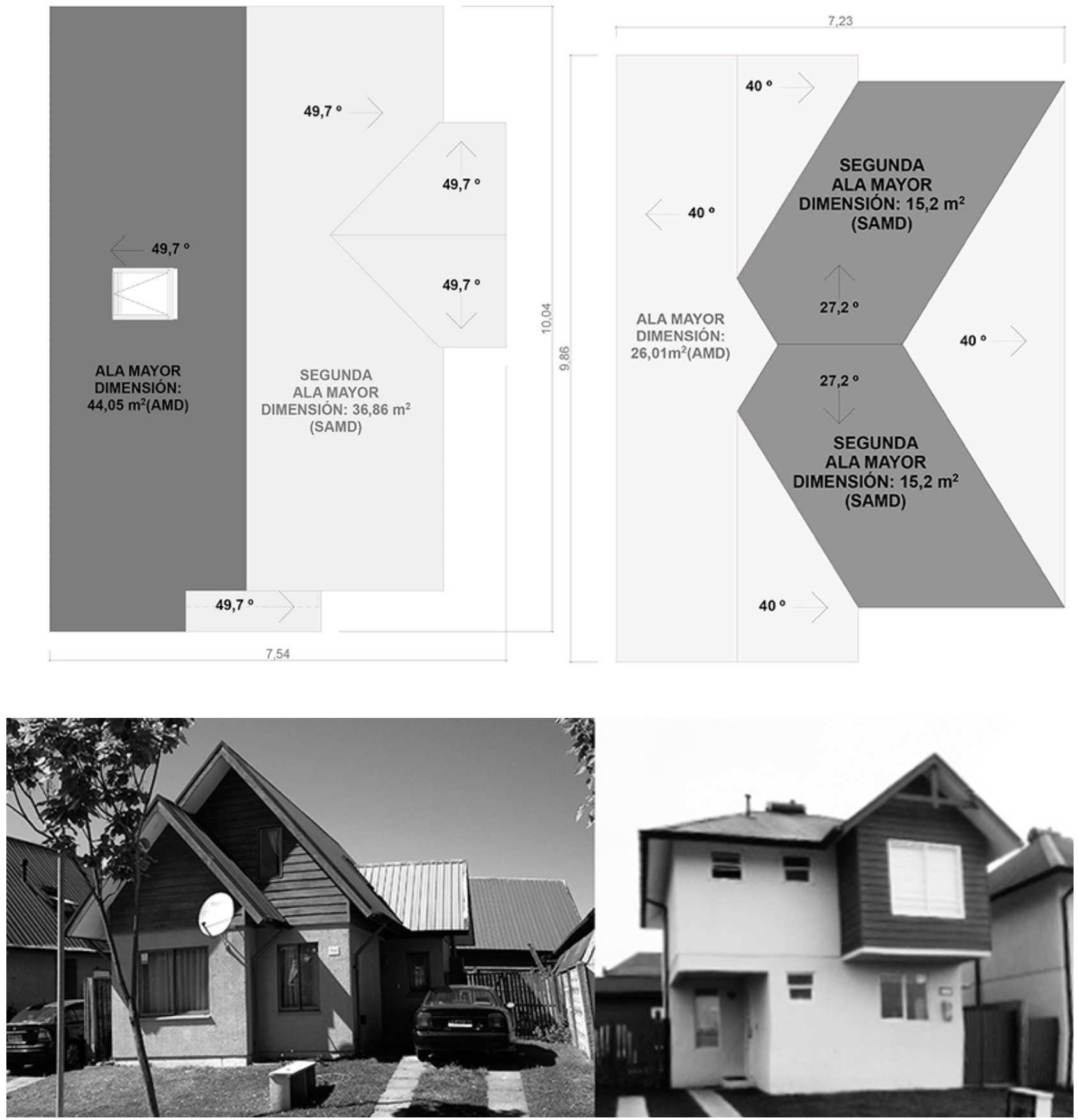

Figura 1. Viviendas en emprendimientos inmobiliarios y Ala de Mayor Dimensión (derecha) y dos Segundas Alas de Mayor Dimensión (izquierda). Fuente: Elaboración propia.

$$
\begin{aligned}
R_{G m_{-} \text {inci-i }}= & R_{G m_{-}} \times F_{d_{1}} \times\left[1-(3,5 / 100000) \times O_{P S}\right] \times \\
& {[1-(P S / 100)][1] }
\end{aligned}
$$

\section{Donde:}

$R_{G m \text { inc-i }}$ Radiación solar global media mensual sobre superficie inclinada del mes $i\left[\mathrm{kWh} / \mathrm{m}^{2}\right]$.

$R_{G m}:$ Radiación solar global sobre superficie horizontal media mensual para el mes $i$ en $[\mathrm{kWh} / \mathrm{m} 2]$.
$F_{d}:$ :Factor modificador de la radiación incidente en una superficie inclinada para el mes $i$. Este valor se obtiene del Anexo II de la Ley 20365, conforme a la inclinación y a la latitud media de la comuna correspondiente.

OpS: Orientación del plano solar. PS: Pérdidas por sombra [\%].

Las pérdidas por sombra, así como por topografía o bloqueo por vegetación, se consideran de forma estadística de acuerdo a estimaciones provenientes de otros contextos (Pelland y Poissant, 2006). 


\section{RESULTADOS.}

\section{DIMENSIONAMIENTO DE CONJUNTOS HABITACIONALES DE VIVIENDAS UNIFAMILIARES.}

Las viviendas unifamiliares ocupan una superficie horizontal considerable, seleccionable y trazable. Se detecta un crecimiento de ciudad de 906.783,4 $\mathrm{m}^{2}$ solamente en emprendimientos residenciales unifamiliares. Se identifican modelos individuales a través de un sistema de Catastro Municipal Electrónico de la Municipalidad (Dirección de Obras Municipales de Concepción, 2015) y se obtienen datos de más de la mitad de los conjuntos de las casas, que ocupan $633.350,1 \mathrm{~m}^{2}$, el 69,8\% de un total de 22 conjuntos detectados.

La casa promedio posee $289,47 \mathrm{~m}^{2}$ en superficie de ocupación de ciudad, correspondiente al lote propio más las vías y cuotas de espacio público respectivos. El crecimiento entre 2006 y 2013 es proyectado en 3.133 casas, correspondiente al 90,6\% de permisos emitidos, de acuerdo al Observatorio Urbano de Chile (3.458 licencias) (Observatorio Habitacional, 2015). El 9,4\% restante se refiere a unidades construidas fuera de conjuntos habitacionales, o bien, aprobados no edificados (Figura 2). Las 2.139 casas efectivamente analizadas representan el $3,3 \%$ frente a la totalidad de viviendas existentes (65.626, según CASEN, 2009), y el 4,1\% respecto a la tipología casa (52.630 unidades registradas legalmente). No es una muestra representativa de la totalidad de la tipología "Casa de Concepción", porque los modelos en emprendimientos unifamiliares presentan diferencias en relación a tipologías anteriores, pero sí lo es de la que se ejecuta en la actualidad. Respecto a la representatividad del universo de estudio, se espera un error del 1,1\% y una confianza del $95 \%$. Asimismo, se encuentran superficies construidas variables entre $57 \mathrm{~m}^{2}$ hasta $170 \mathrm{~m}^{2}$, con un promedio de 93,32 $\mathrm{m}^{2}$; dimensión superior a la estadística nacional (CCHC, 2014), pero cercana a la observada para la ciudad (MINVU, 2013). Casi la totalidad de las viviendas poseen dos plantas $(98,7 \%)$, solo 28 poseen tres. Presentan sistemas constructivos similares entre sí, todas las plantas bajas son de albañilería y el segundo nivel es mayoritariamente de estructura liviana de madera o metal liviano galvanizado semejante a lo descrito por otros autores (Celis et al., 2012)

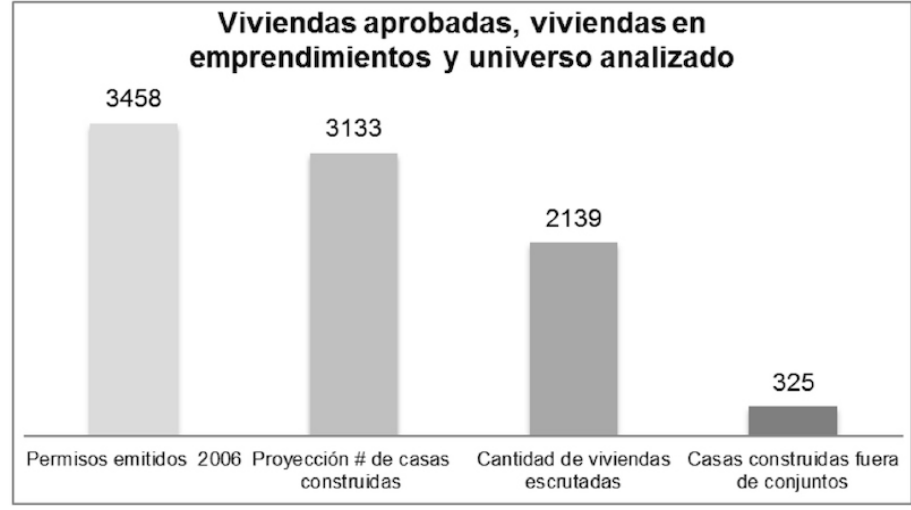

Figura 2. Representatividad de vivienda unifamiliar en emprendimientos inmobiliarios y sujeto de estudio frente a viviendas unifamiliares aprobadas entre 2006 y 2013. Fuente: Elaboración propia.

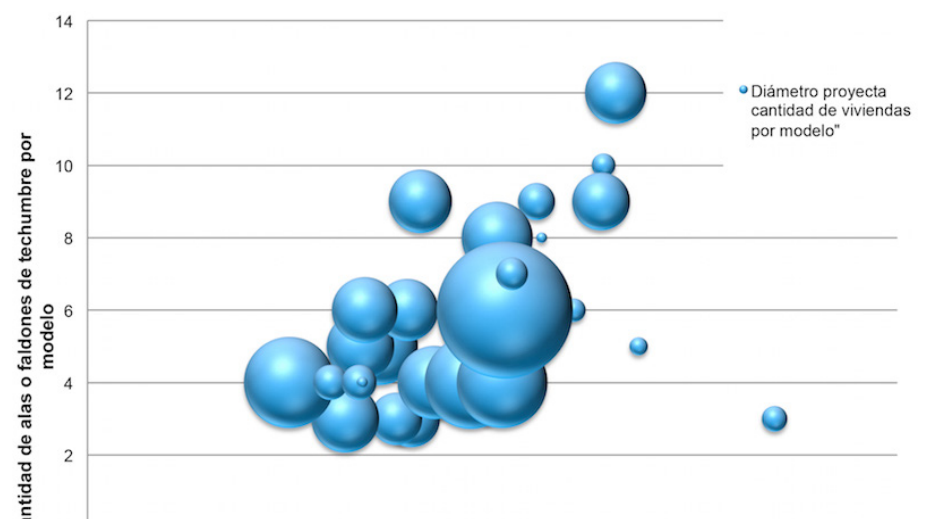

Figura 3. Relación de cantidad de alas de techumbre frente a superficie de vivienda y cantidad de viviendas por modelo. Fuente: Elaboración propia.

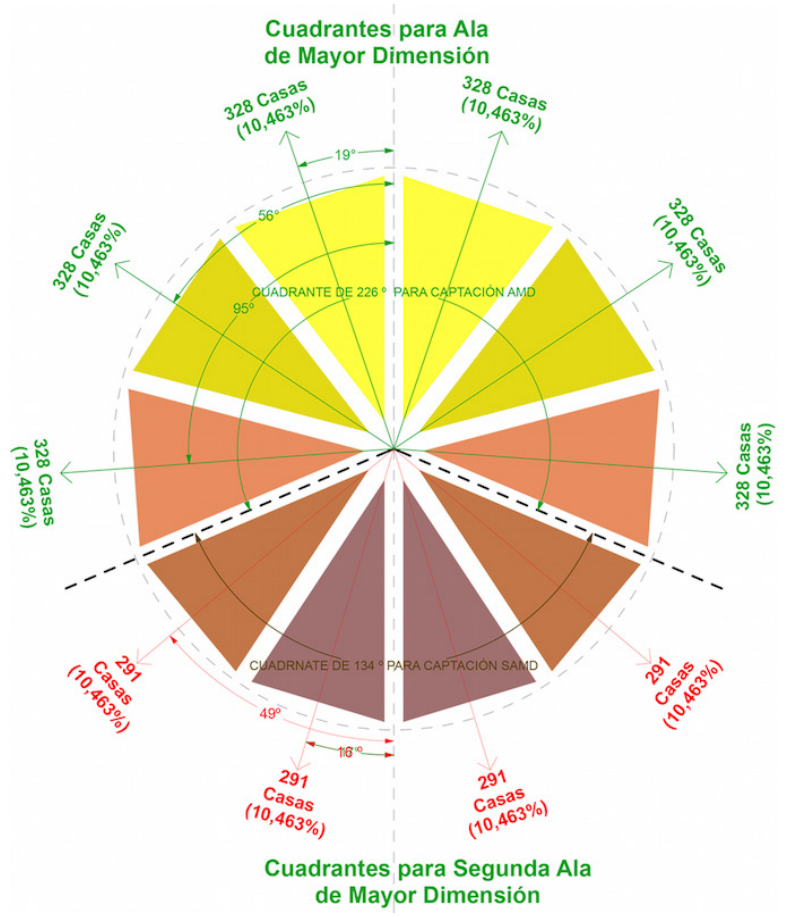

Figura 4. Distribución de número de casas de universo construido a partir de 2006 en emprendimientos inmobiliarios masivo, como representación de orientaciones probables de ala de techumbre captadora (AMD y SAMD). Fuente: Elaboración propia. 


\section{CARACTERIZACIÓN DE TECHUMBRES.}

La proyección plana de techos ocupa $209.479,9 \mathrm{~m}^{2}$ o el $23 \%$ de la superficie total de los loteos; lo restante corresponde a jardines, vías, espacios públicos o terrazas. Al calcularse como superficie inclinada, esta resulta obviamente superior a la horizontal en 129\%. Las techumbres poseen entre 3 y 12 faldones de cubierta por casa, pero la mayor recurrencia, en ese sentido, se encuentra entre los 3 y 6 (Figura 2). A mayor tamaño de casa, la tendencia es tener techumbres más facetadas. La media, considerando todos los casos, está entre 5 y 6 faldones.

Se advierten dos pendientes moda, de $40,0^{\circ}$ y $26,71^{\circ}$, utilizadas por 1000 casos (47\%). El promedio global es de $37,5^{\circ}$, cercana al óptimo para recolección fotovoltaica. El rango máximo y mínimo de inclinaciones es de $19,7^{\circ}$ hasta $49,7^{\circ}$. El $54,7 \%$ de casos posee inclinación superior a la latitud (entre $40^{\circ}$ y $49^{\circ}$ ), condición adecuada para la recolección de energía térmica útil (Gajbert, 2008; Munari, 2009).

\section{DETERMINACIÓN DE AMD, SAMD Y AZIMUT.}

El potencial energético solar está en relación directa y lineal con la superficie captadora disponible: a mayor superficie, mayor producción. Emprendimientos con criterio de aprovechamiento solar-activo presentan generalmente amplios paños y correcta orientación (IEA, 2009; Hachem, 2012). Los diagramas tridimensionales permiten encontrar el AMD y SAMD por caso. Al relacionar la superficie captadora con la superficie construida, se detecta el potencial por modelo, que en la mayoría de casos está entre 0,25 y 0,45, casos extremos muestran 0,20 como mínimo y 0,62 como máximo. El promedio aquí, de 0,39 (Figura 3), indica una potencial superficie colectora de $1.222 \mathrm{~m}^{2}$ en las 3.133 unidades, lo cual representa 9,1 $\mathrm{m}^{2}$ por habitante; frente a ello Izquierdo, Rodrigues y Fueyo (2008) determinaron, para España, una superficie colectora de $14 \mathrm{~m}^{2}$ por habitante, no obstante, adoptaron una superficie de techumbre en proyección plana y en diversas tipologías, sin tener en cuenta los faldones y su aptitud. La relación entre AMD frente a la superficie de ciudad es 0,13.

Los análisis de los loteos evidencian que existe una orientación anárquica de faldones de techumbre, de hecho AMD o SAMD pueden encarar hacia delante, detrás o a los costados; además, los loteos y su consecuente orientación tampoco llevan ninguna relación respecto a orientación de faldones. En consecuencia, se supone igual posibilidad de orientación para cualquiera de los puntos cardinales.

\section{ESTIMACIÓN DE IRRADIACIÓN SOLAR INCIDENTE EN TECHUMBRES INCLINADAS Y PRODUCCIÓN ELÉCTRICA PV.}

Para determinar el potencial solar, se dimensiona la irradiación anual que recibe el faldón AMD o SAMD. Cuando AMD se orienta desfavorablemente, SAMD está en dirección opuesta o perpendicular mejor dispuesta, en consecuencia. Se calcula la condición promedio cuando el SAMD es más favorable. Y se estima la irradiación acorde a la inclinación y orientación frente a la relación promedio de $77,24 \%$, que corresponde al promedio de superficie SAMD respecto a AMD. Se utilizan, entonces, dos inclinaciones representativas para el cálculo, las que son resultado de las dos pendientes moda. El primer grupo representa pendientes pronunciadas que engloban el $54,4 \%$ de los casos (1.163 viviendas), cuyo promedio es de $43,7^{\circ}$; mientras que el segundo grupo contiene el $45,6 \%$ (976 casos) restante y una inclinación promedio de $26,8^{\circ}$.

La irradiación disponible se obtiene aplicando el modelo [1] descrito en el Apartado 2 de este documento. En primera instancia, no se consideran las "Pérdidas por Sombras" (PS), ya que esta variable debería analizarse para cada caso puntual. Sin embargo, se estipulan pérdidas conjuntas estadísticamente posibles, a partir de estudios referentes en viviendas de similares características que incorporan captación activa por condiciones de sombras, limpieza, mantenimiento, etc. (Pelland y Poissant, 2006). El cálculo de potencial captación por orientación en inclinación establece que cuando AMD está desviada $113,0^{\circ}$ respecto al norte verdadero, la irradiación captada es similar a la SAMD con una rotación de $90^{\circ}$ respecto al norte (considerando las dos pendientes promedio), por lo tanto, se adopta como desviación estadística para, con desviación superior a $113^{\circ}$, empezar a utilizar el indicador SAMD. En consecuencia, se reparte el universo de casas distributivamente con $A M D$, captando dentro del rango de $226^{\circ}$ con dirección norte ( $113^{\circ}$ desvío al este y oeste), grupo al que corresponde a 1.967 casas. Luego, para las unidades restantes se supone captación con SAMD, encarando dentro del ángulo de $139^{\circ}$ restantes siendo en un número de 1166 (Figura 4).

En la misma Figura 4, el cuadrante de $226^{\circ}$ que se representa en la zona superior de la distribución, muestra seis subcuadrantes correspondientes a seis grupos con desviaciones de AMD; y el cuadrante de SAMD, en zona inferior, de $134^{\circ}$, se subdivide en cuatro. De este modo, los cuadrantes AMD y SAMD presentan similar cantidad de casas (328, las AMD y 292, las SAMD). Los seis subcuadrantes superiores de AMD configuran un ángulo de $37,2^{\circ}$, los de SAMD, ángulos de $33,5^{\circ}$. Para calcular la irradiación solar, la orientación este y oeste se suponen similares, cuantificándose conjuntamente. Así, la fracción con captación AMD incluye 656 casas con desviación de 
IRRADIACIÓN SOLAR ANUAL (MGWh)EN AMD O SAMD Y POTENCIAL PV ESTADÍSTICAMENTE DETERMINADO

\begin{tabular}{|c|c|c|c|c|c|c|c|c|c|c|c|c|c|c|c|c|c|}
\hline $\begin{array}{c}\text { Desvío en } \\
\text { Azimunth(을 de } \\
\text { captación }\end{array}$ & Inclinación (ㅇ) & $\begin{array}{c}\text { \# de } \\
\text { viviendas }\end{array}$ & $\begin{array}{c}\text { AMD o } \\
\text { SAMD (m2) }\end{array}$ & Ene & Feb & Mar & Apr & May & Jun & Jul & Ago & Sep & Oct & Nov & Dic & $\begin{array}{c}\text { Promedi } \\
\text { o Anual } \\
\end{array}$ & TOTAL \\
\hline & $\begin{array}{c}26.8 \text { ( } 45.6 \% \text { de } \\
655 \text { casos) } \\
43.7(54.4 \% \text { de }\end{array}$ & 299 & 37,24 & $2.263,8$ & $1.852,2$ & $1.841,1$ & $1.399,5$ & 905,8 & 765,2 & 813,6 & $1.134,8$ & $1.453,3$ & $1.870,4$ & $1.983,5$ & $2.135,4$ & $1.534,9$ & $18.418,6$ \\
\hline 19,1 & $\begin{array}{c}656 \text { casos }) \\
26.8(45.6 \% \text { de }\end{array}$ & 356 & 37,24 & $2.444,6$ & $2.122,2$ & $2.250,4$ & $1.804,5$ & $1.233,5$ & $1.071,4$ & $1.119,5$ & $1.502,5$ & $1.803,3$ & $2.166,3$ & $2.168,6$ & $2.258,8$ & $1.828,8$ & $21.945,7$ \\
\hline 57,50 & $\begin{array}{c}655 \text { casos) } \\
43.7 \text { (54.4\% de }\end{array}$ & 299 & 37,24 & $2.027,9$ & $1.659,2$ & $1.649,3$ & $1.253,6$ & 811,4 & 685,5 & 728,8 & $1.016,6$ & $1.301,8$ & $1.675,5$ & $1.776,9$ & $1.912,9$ & $1.375,0$ & $16.499,4$ \\
\hline & $\begin{array}{c}656 \text { casos }) \\
26.8(45.6 \% \text { de }\end{array}$ & 356 & 37,24 & $2.189,9$ & $1.901,1$ & $2.015,9$ & $1.616,5$ & $1.105,0$ & 959,8 & $1.002,9$ & $1.345,9$ & $1.615,4$ & $1.940,5$ & $1.942,7$ & $2.023,5$ & $1.638,3$ & $19.659,0$ \\
\hline 95,83 & $\begin{array}{c}655 \text { casos) } \\
43.7 \text { (54.4\% de }\end{array}$ & 299 & 37,24 & $2.004,3$ & $1.640,0$ & $1.630,1$ & $1.239,1$ & 802,0 & 677,5 & 720,3 & $1.004,8$ & $1.286,7$ & $1.656,0$ & $1.756,2$ & $1.890,7$ & $1.359,0$ & $16.307,6$ \\
\hline & $\begin{array}{c}656 \text { casos }) \\
26.8(48.72 \% \text { of }\end{array}$ & 356 & 37,24 & $2.164,4$ & $1.879,0$ & $1.992,4$ & $1.597,7$ & $1.092,1$ & 948,6 & 991,2 & $1.330,3$ & $1.596,6$ & $1.918,0$ & $1.920,1$ & $1.999,9$ & $1.619,2$ & $19.430,4$ \\
\hline 16,24 con SAMD & $\begin{array}{c}583 \text { casos }) \\
43.7(51.28 \% \text { of }\end{array}$ & 266 & 28,28 & $1.534,4$ & $1.255,5$ & $1.247,9$ & 948,6 & 613,9 & 518,7 & 551,4 & 769,2 & 985,0 & $1.267,8$ & $1.344,4$ & $1.447,4$ & $1.040,4$ & $12.484,2$ \\
\hline & $\begin{array}{c}583 \text { cases }) \\
26.8(48.72 \% \text { of }\end{array}$ & 317 & 28,28 & $1.657,0$ & $1.438,5$ & $1.525,3$ & $1.223,1$ & 836,1 & 726,2 & 758,8 & $1.018,4$ & $1.222,3$ & $1.468,3$ & $1.469,9$ & $1.531,1$ & $1.239,6$ & $14.874,9$ \\
\hline AMD & 583 cases) & 266 & 28,28 & $1.419,9$ & $1.161,8$ & $1.154,8$ & 877,8 & 568,1 & 480,0 & 510,3 & 711,8 & 911,5 & $1.173,1$ & $1.244,1$ & $1.339,4$ & 962,7 & $11.552,4$ \\
\hline & $\begin{array}{c}43.7 \text { ( } 51.28 \% \text { of } \\
583 \text { cases) }\end{array}$ & 317 & 28,28 & $1.533,3$ & $1.331,1$ & $1.411,5$ & $1.131,8$ & 773,7 & 672,0 & 702,2 & 942,4 & $1.131,0$ & $1.358,7$ & $1.360,2$ & $1.416,8$ & $1.147,1$ & $13.764,7$ \\
\hline \multicolumn{2}{|c|}{ TOTAL } & 3133 & & $19.239,6$ & $16.240,6$ & $16.718,7$ & $13.092,0$ & $8.741,5$ & $7.504,8$ & $7.899,1$ & $10.776,6$ & $13.306,9$ & $16.494,6$ & $16.966,6$ & $17.956,0$ & $13.744,7$ & $164.937,0$ \\
\hline \multicolumn{18}{|c|}{ Potencial Eléctrico PV (IEA/ PELLAND Y POISSANTE / } \\
\hline
\end{tabular}

Tabla 1. Cálculo de irradiaciones en grupos de casas en indicadores AMD y SAMD, mensual y anualmente. Fuente: Elaboración propia.

$19,10^{\circ}, 57,5^{\circ}$ y $95,83^{\circ}$ respecto al norte; en tanto, el grupo SAMD resulta en 583,0 casos con desviación de $16,24^{\circ}$ y $48,75^{\circ}$. Con la Fórmula [1] se estima la irradiación recibida en las techumbres por cada grupo, tanto AMD como SAMD, de manera mensual y con ello se consolida en producción conjunta de la totalidad de casas en margen anual.

La Tabla 1 muestra la irradiación total conjunta, oscilaciones acorde a inclinación promedio y por número. Lógicamente, el grupo que exhibe máxima producción con captación AMD es aquel con menor desviación $\left(19,1^{\circ}\right)$ e inclinación de $43,7^{\circ}$, el cual recibe irradiación conjunta de $21945,7 \mathrm{MWh}$ al año. El grupo con menor producción utiliza su SAMD con alta desviación $\left(48,75^{\circ}\right)$ e inclinación de $26,8^{\circ}$, captando anualmente 11.552,4 MWh. El mes con máxima irradiación conjunta es enero, con 19.239,0 MWh; junio posee la mínima, con 7.504,8 MWh. La producción energética en el conjunto de casas con captación AMD y desvío de $95,8^{\circ}$ es superior a aquellas que utilizan la SAMD desviadas $16,24^{\circ}$ (aunque también son más numerosas). Se observa escasa diferencia de irradiación promedio anual por $\mathrm{m}^{2}$ entre techos inclinados $43,7^{\circ}$ y con inclinación de $26,8^{\circ}$, aunque mensualmente se adviertan mayores distinciones.

Con la irradiación obtenida se puede prever generación eléctrica PV. La literatura especializada presenta una eficiencia de $h=16 \%$ como un válido promedio para fotovoltaicos instalados en centros urbanos conectados a red, no obstante, este potencial debe castigarse, por pérdidas estadísticamente determinadas por inversores, distribución, sombreados, factores de conexión, etc., en un porcentaje del 25\% (Pelland y Poissant, 2006). Se espera entonces una producción útil del 12\% respecto a la irradiación recibida. Esta estadística es obtenida con redes, instalaciones, tecnología y condiciones adecuadas en comunidades que han adoptado la recolección solar como política de estado (IEA, 2009), y podría esperarse en el caso chileno solamente si se siguieran similares impulsos, facilidades, conexiones y distribución de excedentes.

La Tabla 1 también expresa la producción eléctrica estimada en las 3.133 techumbres de 19.792,4 MWh anualmente, lo que supone $6.317,4 \mathrm{KWh}$ por casa en promedio. Al expresarse por superficie construida y casa promedio de $93,32 \mathrm{~m}^{2}$, se puede inferir una producción de $67,71 \mathrm{KWh}$ de electricidad anual por $\mathrm{m}^{2}$ habitable. La superficie de techumbre productora total es $106.197,4 \mathrm{~m}^{2}$, lo cual significa una producción anual de 186,4 KWh por m² de techumbre recolectora al año. 


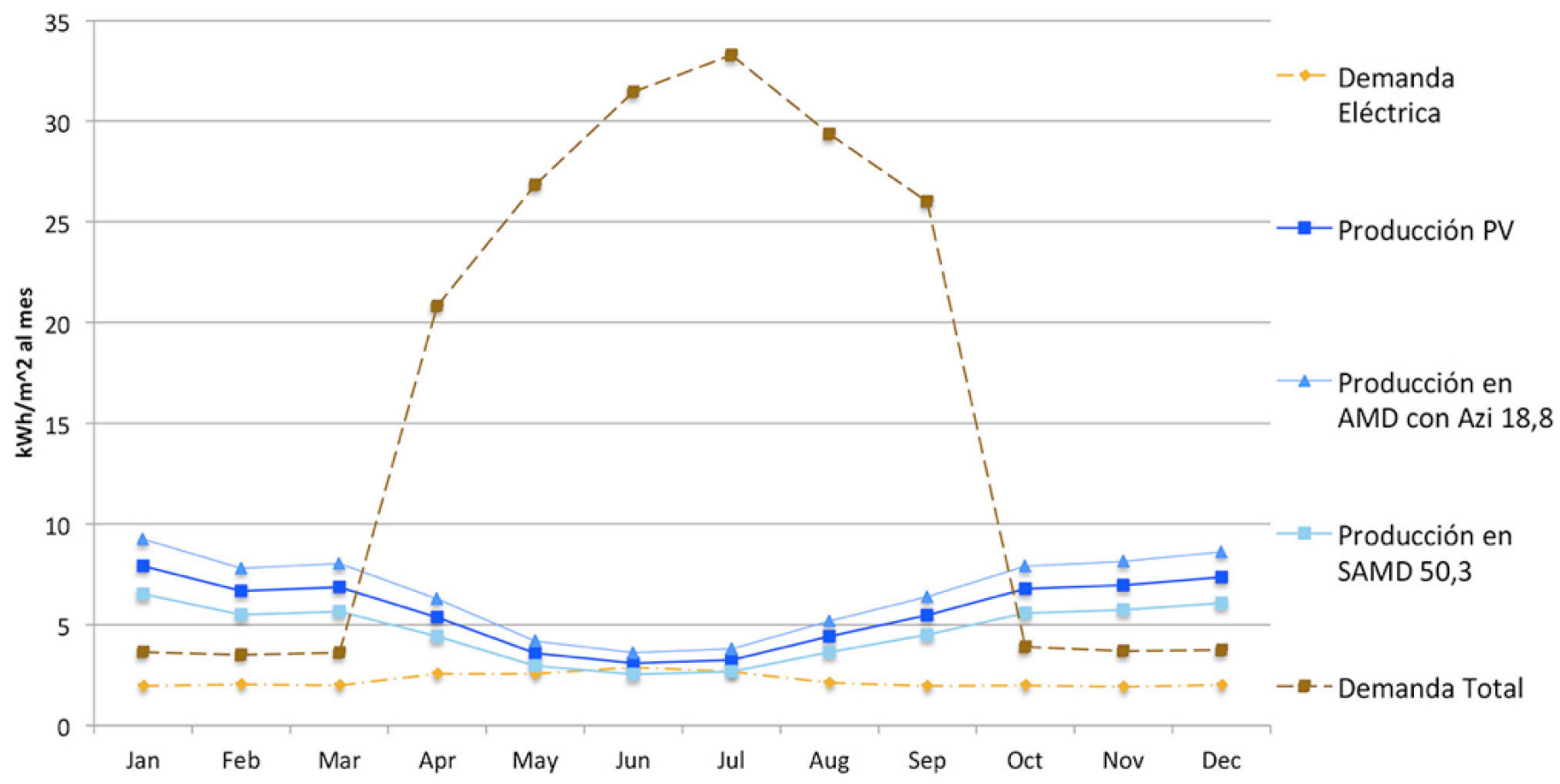

Figura 5. Curvas Promedio de demanda eléctrica y energética global frente a producción promedio por m2 de superficie construida. Fuente: Elaboración propia.

\section{COMPARACIÓN ENTRE POSIBLE PRODUCCIÓN ENERGÉTICA SOLAR FRENTE A DEMANDA ELÉCTRICA RESIDENCIAL Y ALTERNATIVA HÍBRIDA TÉRMICA-FOTOVOLTAICA.}

La producción eléctrica estimada temporalmente es comparada con la demanda determinada estadísticamente para Concepción (CDT 2010) en la Figura 5. La principal limitación para un abastecimiento total es la característica alta demanda invernal frente a la baja producción por la mínima irradiación existente en tal temporada. De forma complementaria, se observan posibilidades extremas acordes a tipología de casa, al analizar el caso con mayor proporción AMD por superficie habitable y orientado prácticamente al norte $\left(18,83^{\circ}\right.$ de desviación), frente a caso con utilización SAMD encarando noreste y noroeste con alta desviación $\left(50,25^{\circ}\right)$. Los resultados muestran que en época estival se supera el autoabastecimiento para la demanda eléctrica con amplio superávit; pero en el caso extremo desfavorable no se alcanza (aunque por un escaso margen) a suplir la demanda de la época invernal. En todos los casos, durante el verano existe alto margen de energía exportable para abastecer otras demandas en la ciudad (Lund, 2012). En cuanto a la demanda residencial total, se aprecia un marcado desbalance en los meses fríos, por calefacción; los estudios sugieren reducir la demanda de energía térmica a través de medidas de eficiencia energética pasiva, en primera instancia, luego, la captación de energía térmica solar de forma activa es una opción que puede contribuir efectivamente a disminuir el desbalance (Charron y Athienitis, 2006; Hastings y Wall, 2007).

La gráfica de la Figura 5 expone la relación de los casos extremos, aquel con mayor proporción AMD/superficie construida y aquel modelo con inferior potencial y orientación, en términos de producción-demanda mensual. En suma, se estipula un índice de producción máxima de $78,9 \mathrm{kWh}$ al año, frente a 55,3 kWh al año como mínimo expresado en indicadores por $\mathrm{m}^{2}$ de vivienda habitable.

La alta demanda térmica impulsa a explorar alternativas como una posible captación térmica con Colectores híbridos Fotovoltaicos/Térmicos con fluido aire (BIPVT/a). Esta tecnología ya comercializada en Canadá es una alternativa con oportunidades interesantes ya que optimiza la superficie de captación, al tiempo que evita el riesgo por sobreproducción estival y mejora la producción eléctrica (Solarwall, 2015; Chow, 2010; Kumar y Rosen, 2011). Del total de irradiación absorbida por los paneles, aproximadamente el $85 \%$ se disipa en forma de calor, temperatura adicional que provoca baja de producción eléctrica. En ese sentido, se esperan pérdidas de 0,4\% en eficiencia por ${ }^{\circ} \mathrm{C}$ de incremento de temperatura (Astea, Del Peroa y Leonfortea, 2012; Hachem 2012). La tecnología híbrida con aire supone paneles fotovoltaicos sobrepuestos a una cámara de circulación de aire para refrigerar las células, de manera que el calor recuperado puede utilizarse de ser requerido, conduciéndolo al interior. Hachem (2012) relaciona la producción de electricidad y calor útil generados en techumbres PVTa con inclinación 


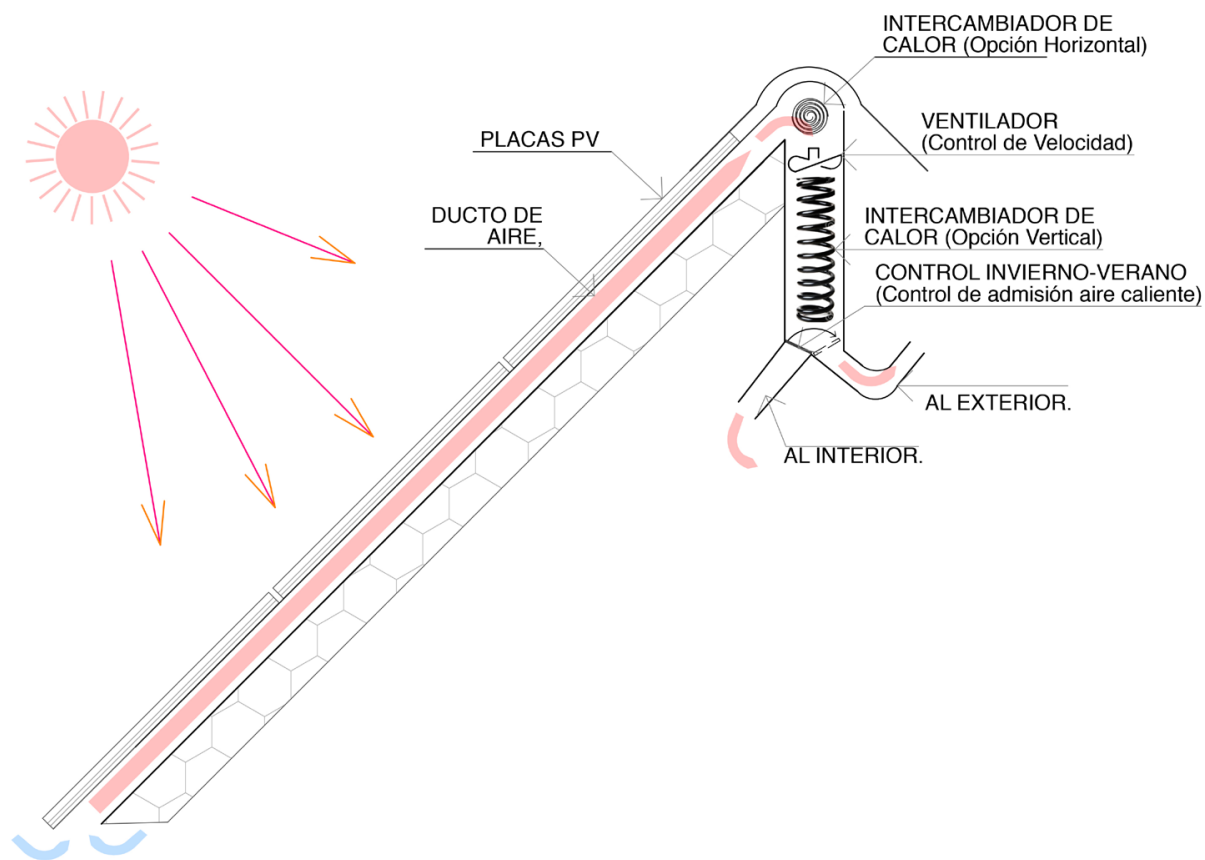

Figura 6: Esquema de detalle de funcionamiento de tecnología BIPVTa. Fuente: Hachem, 2012; Solarwall, 2015.

\section{Abastecimiento esperado máximo y minimo: $74,6 \%$ y 41,3\%, Abastecimiento esperado máximo y minimo: $138,6 \%$ y $107,7 \%$}

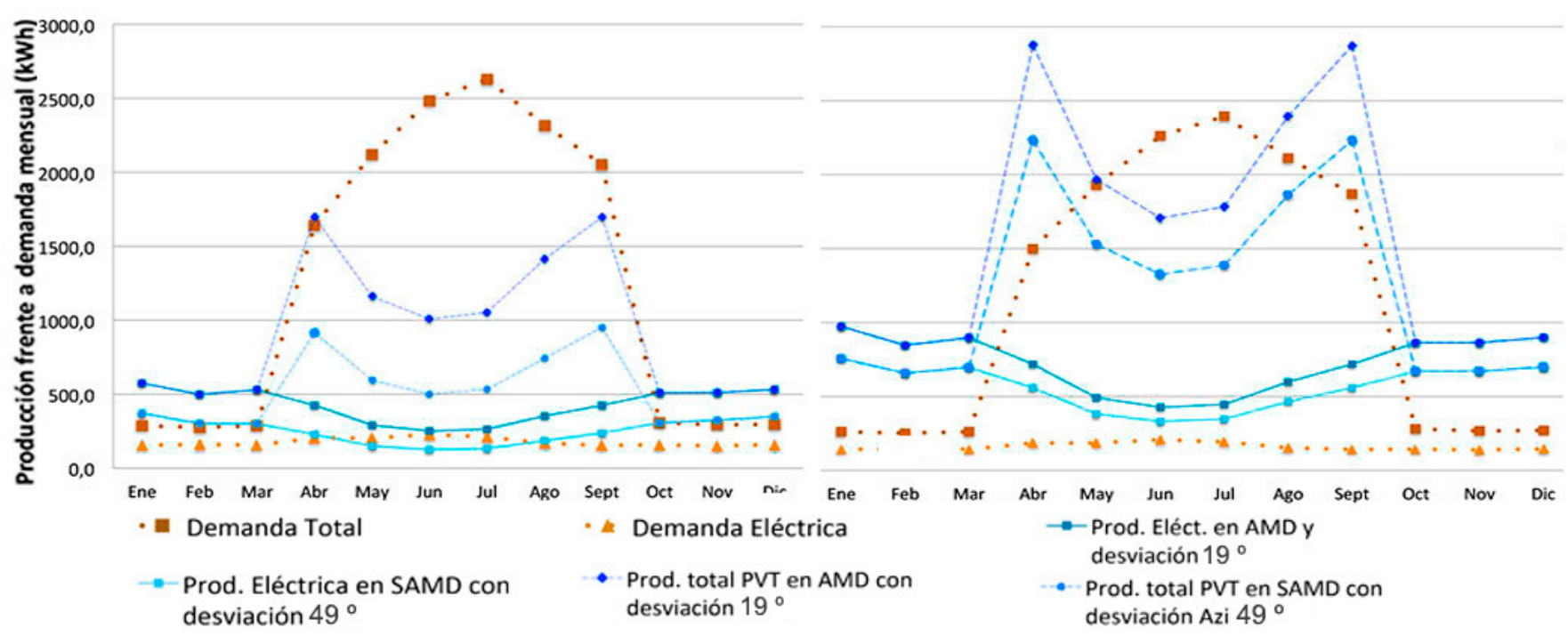

Figura 7. Producción-Demanda mensual en viviendas con SAMD y AMD extremos. Fuente: Elaboración propia 
entre $30^{\circ}$ y $60^{\circ}$, tomando como condición principal una inclinación de $45^{\circ}$. Siendo $Q_{e}$ la energía eléctrica generada y $Q_{u}$ el total de energía térmica, los estudios recién citados muestran una relación $Q_{u} / Q_{e}=3,0$ a 3,5 con condiciones adecuadas de velocidad de aire; y finalmente adoptan la condición $3 Q_{u}=Q_{e}$. Se trata de un útil modelo simplificado para sistemas híbridos con aire para estimación. Cada instalación bajo condiciones de producción y demanda precisas tiene que ser revisada. La energía térmica desechada es trasladada en aire precalentado al interior de la vivienda cuando se requiere, lo cual da como resultado una disminución en la demanda de calefacción. Para la época estival se considera que el aire refrigerante es evacuado al ambiente o utilizado para el calentamiento de agua de uso sanitario a través de un intercambiador de calor aire-agua (Figura 6).

Con los márgenes mencionados, la tecnología híbrida puede aportar significativamente en los meses próximos al equinoccio (marzo-mayo y agosto-octubre) y contribuir marginalmente en invierno. El índice de producción promedio de todos los casos se estima en 142,6 kWh por $\mathrm{m}^{2}$ de energía útil al año (incluyendo electricidad exportable). Respecto a la demanda promedio de 189,9 $\mathrm{kWh} / \mathrm{m}^{2}$, el margen de autoabastecimiento es de $75 \%$ en el caso promedio de vivienda $\left(93,3 \mathrm{~m}^{2}\right)$, en superficie captadora entre 36,8 y 28,4 de AMD y SAMD.

En casos extremos (Figura 1) -el modelo con mayor relación AMD por superficie construida y baja desviación $\left(19^{\circ}\right)$, frente a modelo con inferior potencial utilizando SAMD con alta desviación $\left(49^{\circ}\right)$-, la producción del modelo superior abastecería alrededor del $92 \%$ de la demanda total, aunque el gráfico denota que parte de la producción está sobre la curva de demandas en abril. Por lo tanto, en tal caso puede esperarse un margen de entre $85 \%$ a $90 \%$ de la demanda total. El modelo con inferior condición abastece solamente el $41,3 \%$ de ella. Para cubrir la demanda eléctrica únicamente, se prevé, para el modelo superior, un autoabastecimiento de 317,1\% anual frente al modelo inferior que se abastecería en 148,7\% (Figura 7).

\section{CONCLUSIONES.}

Este trabajo entrega un método para pre-dimensionar, a escala urbana y en viviendas de emprendimientos masivos, la producción eléctrica fotovoltaica y térmica mediante indicadores de rendimiento estadísticos e indicadores de conjuntos de casas tipológicamente seriadas. El procedimiento también trabaja con la disposición y geometría de techumbres recurrentes a partir de supuestos generales de producción fotovoltaica y térmica más fotovoltaica.

Con este método se encuentra el potencial para generación eléctrica de un conjunto de viviendas unifamiliares, al cubrirse de fotocélulas su Ala de Mayor Dimensión (AMD) o su Segunda Ala de Mayor Dimensión (SAMD), según corresponda por orientación. Así, se determina que pueden generarse en total cerca de 19.792,4 MW al año, en una superficie urbana residencial de 906.783,38 $\mathrm{m}^{2}$, a razón de $21,82 \mathrm{~kW}$ por $\mathrm{m}^{2}$. Esto permite abastecer eléctricamente 2,5 veces el consumo total de las viviendas. En relación a la producción térmica más eléctrica, con colectores híbridos, se calcula un posible abastecimiento del 75,1\% del total de energía doméstica, térmica y eléctrica conjunta con la actual configuración de techos.

En los casos extremos del conjunto analizado, al comparar demanda-abastecimiento, la vivienda con mayor potencial de captación AMD y desviación mínima (19 respecto al norte), comparado con el ejemplar con captación en SAMD y alta desviación (49 ${ }^{\circ}$ ), se alcanza un abastecimiento eléctrico del $317,1 \%$ en el más favorable, y $148,7 \%$ el menos favorable. Es decir, todo el rango de viviendas puede captar una cantidad mayor que el propio consumo, y utilizarla con distribución local. En cuanto a demanda energética total, se puede cubrir desde el $43 \%$ (caso menor potencial) hasta entre 108 a139\% (caso superior potencial) aunque dependiente de adecuados sistema de almacenamiento, a través de recolección PVT híbrida y fluido de aire. No se toma en cuenta la posible utilización del exceso térmico estival, que podría ser aplicado en calentamiento de agua caliente sanitaria a través de intercambiador de calor, que podría incrementar el margen de abastecimiento térmico calculado.

Los datos entregados son generales y referenciales, a partir de demandas promedio esperadas. Las condiciones climáticas locales, más las características constructivas promedio, proporcionan estimados que sugieren un alto potencial, por lo que surge la necesidad de una exploración más detallada. Se requiere, en síntesis, de un análisis exhaustivo que disponga de simulaciones precisas, de sistemas constructivos puntuales, de información sobre la demanda y de tecnología de recolección solar específica, además de la construcción de prototipos que validen los resultados del presente estudio. 


\section{AGRADECIMIENTOS.}

Este trabajo es parte de programa de investigación Conicyt CONFIN AKA-ERNC 007, (Chile). Agradecemos también a la Secretaria de Educación Superior, Ciencia, Tecnología e Innovación del Ecuador, SENESCYT y a la Universidad de Cuenca.

\section{REFERENCIAS.}

ASTEA, Niccolò; DEL PEROA, Claudio y LEONFORTEA, Fabrizio. Optimization of solar thermal fraction in PVT systems. Energy Procedia [en línea], 2012, vol. 30, pp. 8-18. [Consultado 15 mayo 2016]. DOI 10.1016/j.egypro.2012.11.003.

CÁMARA CHILENA DE LA CONSTRUCCIÓN (CCHC). Balance de la Vivienda en Chile [en línea]. Santiago de Chile, 2014. [Consultado 9 mayo 2016]. Disponible en: http://www.cchc.cl/ uploads/archivos/archivos/Balance-de-la-Vivienda-2014.pdf

CASEN. Reportes Estadísticos Comunales [en línea], 2009. [Consultado 9 mayo 2016]. Disponible en: http:// reportescomunales.bcn.cl/2012/index.php/Concepción\#Poblaci. C3.B3n_total_2002_y_proyectada_2012_INE

CORPORACIÓN DE DESARROLLO TECNOLÓGICO (CDT). Sistemas Solares Térmicos [en línea], CDT, 2007. [Consultado 15 mayo 2016]. Disponible en: http://informatica.cdt.cl/documentos/ publicaciones/index.php?opcion=publicaciones

CORPORACIÓN DE DESARROLLO TECNOLÓGICO (CDT). Estudio de Usos Finales y Curva de Oferta de Conservación de la Energía en el Sector Residencial de Chile. Santiago de Chile, 2010 .

CELIS, Flavio; GARCÍA, Rodrigo; TREBILCOCK, Maureen; ESCORCIA, Olavo y DÍAZ, Muriel. Análisis energético de las viviendas del centro-sur de Chile. Arquiteturarevista, 2012, vol. 8, pp. 62-75.

CHARRON, Rémi y ATHIENITIS, Andreas. Design and Optimization of Net Zero Energy Solar Homes (AE). ASHRAE Transaction, 2006, vol. 112, pp. 285-296.

CHOW, Tin Tai. A review on photovoltaic / thermal hybrid solar technology. Applied Energy [en línea], 2010, vol. 87, $n^{\circ} 2$, pp. 365-379. [Consultado 20 mayo 2016].DOI 10.1016/j. apenergy.2009.06.037.

DIRECCIÓN DE OBRAS MUNICIPALES DE CONCEPCIÓN. Sistema de Catastro Municipal Electrónico [en línea], 2015. [Consultado24 octubre2015]. Disponibleen:http://200.68.12.118/ SigDom_comps/SigDom.phtml

GAJBERT, Helena. Solar thermal energy systems for building integration [en línea]. Lund University, 2008. Disponible en: http:// www.ebd.Ith.se/fileadmin/energi_byggnadsdesign/images/ Publikationer/Lic_avhandling_HG_G5_web.pdf

HACHEM, Caroline, Investigation of Design Parameters for Increased Solar Potential of Dwellings and Neighborhoods. Concordia University, 2012.

HASTINGS, S. Robert y WALL, Maria. Sustainable Solar Housing: Volume 2 - Exemplary Buildings and Technologies. London \& Sterling VA: Earthscan, 2007.

INTERNATIONAL ENERGY AGENCY (IEA), Cities, Towns \& Renewable Energy Cities, Towns [en línea]. París: IEA/OECD, 2009. [Consultado 11 abril 2016]. Disponible en: http://www.iea. org/publications/freepublications/publication/Cities2009.pdf 
IEA SHC TASK 41, 2012, Solar Energy in Architecture. Integration criteria and guidelines [online]. Available from: http://leso2.epfl. ch/solar/pdf/SolThePh.pdf

IZQUIERDO, Salvador; RODRIGUES, Marcos y FUEYO, Norberto. A method for estimating the geographical distribution of the available roof surface area for large-scale photovoltaic energypotential evaluations. Solar Energy, 2008. vol. 82, n 10, pp. 929939.

KALOGIROU, Soteris. Solar thermal collectors and applications. Progress in Energy and Combustion Science, 2004, vol. 30, pp. 231-295.

KUMAR, Rakesh y ROSEN, Marca. A critical review of photovoltaicthermal solar collectors for air heating. Applied Energy [en línea], 2011, vol. 88, n 11, pp. 3603-3614. [Consultado 15 mayo 2016]. DOI 10.1016/j.apenergy.2011.04.044.

LUKAČ, Niko y ŽALIK, Borut. GPU-based roofs' solar potential estimation using LiDAR data. Computers and Geosciences, 2013, vol. 52, pp. 34-41.

LUND, Peter. Large-scale urban renewable electricity schemes - Integration and interfacing aspects. Energy Conversion and Management [en línea], 2012, vol. 63, pp. 162-172. [Consultado 4 mayo 2016]. DOI 10.1016/j.enconman.2012.01.037.

MINENERGIA. Norma Técnica que determina algoritmo para la verificación de la contribución solar mínima de los Sistemas Solares Térmicos acogidos a la franquicia tributaria de la Ley $N^{\circ}$ 20.365 [en línea]. 2009. Disponible en: http://antiguo.minenergia. cl/minwww/export/sites/default/02_Noticias/descargas_noticias/ Norma_Tecnica_Actualizada.pdf

MINISTERIO DE VIVIENDA Y URBANISMO (MINVU), Proyectos de Tipo de Viviendas [en línea]. 2012. Available from: http://www. minvu.cl/opensite_20111121172350.aspx

MUNARI, Cristina. Architectural Integration and Design of Solar Thermal Systems. ÉCOLE POLYTECHNIQUE FÉDÉRALE DE LAUSANNE, Switzerland, 2009. [Consultado 9 junio 2016]. Disponible en: http://www.bisolnet.ch/Munari\%20Probst-final.pdf

OBSERVATORIO HABITACIONAL MINVU. Estadísticas/ Variación anual de unidades y superficie de viviendas [en línea]. 2014. [Consultado 15 mayo 2016]. Disponible en: http://www. observatoriohabitacional.cl/opensite_20080122171157.aspx

OBSERVATORIO HABITACIONAL MINVU. Viviendas unidades y superficie según mes y comuna 2002-2013-3 [en línea]. 2015. [Consultado 4 mayo 2016]. Disponible en: http://www. observatoriohabitacional.cl/opensite_20080122171157.aspx

PELLAND, Sophie y POISSANT, Yves. An Evaluation of the Potential of Building Integrated Photovoltaics in Canada. Renewable Energy [en línea], 2006, p. 8. [Consultado 6 marzo 2016]. Disponible en: http://canmetenergy-canmetenergie.nrcanrncan.gc.ca/fichier.php/codectec/En/2006-047/2006-047_OPJ_411-SOLRES_BIPV_new.pdf

SOLARWALL. PV/Thermal; Hybrid Solar Heating + Electricity [en línea]. 2015. [Consultado 3 marzo 2015]. Disponible en: http:// solarwall.com/en/products/pvthermal.phpSolarWall@
WALL, Maria; MUNARI PROBST, Maria Cristina; ROECKER, Christian; DUBOIS, Marie Claude; HORVAT, Miljana; JØRGENSEN, Olaf Bruun y KAPPEL, Karin. Achieving solar energy in architecture - IEA SHC Task 41. Energy Procedia [en línea], 2012, vol. 30, pp. 1250-1260. [Consultado 3 marzo 2015]. Disponible en: http:// www.sciencedirect.com/science/article/pii/S1876610212016542 